\title{
Modality-specific interference with verbal and nonverbal stimulus information
}

\author{
TERRY W. ALLEN, MICHAEL M. MARCELL, and PATRICIA ANDERSON \\ Vanderbilt University, Nashville, Tennessee 37240
}

\begin{abstract}
Subjects recalled both letters and the locations of letters in 2 by 3 and 1 by 6 matrices after either no interfering activity or visual, auditory, or kinesthetic interfering activity. Results for each type of matrix indicated the presence of selective auditory (verbal) interference with the recall of letter identity and selective visual and kinesthetic interference with the recall of letter location. Supplementary correlational analyses indicated that the presence of such a dual encoding strategy was most consistent across subjects for the 2 by 3 matrix. Although the results indicated that use of different modes of representation was related to the verbal-nonverbal nature of the information, it was shown that the structure of the stimulus array also affected the nature of the representation.
\end{abstract}

Early conceptions (e.g., Sperling, 1963, 1967) concerning the nature of representation of information in short-term memory postulated that nonverbal as well as verbal information is encoded and stored in a verbal form. Recent experiments in this area have sought to establish the existence of a separate visual memory code as it relates to the representation of nonverbal information. Moreover, investigators have been interested in determining the extent to which visual and verbal codes are stored separately. In order to examine these issues, investigators have frequently used the retroactive interference paradigm to infer the nature of the representation in memory of a particular type of information. It is assumed in this paradigm that an intervening activity can be used to interfere selectively with the mode of representation employed by an individual.

Several experimenters (den Heyer \& Barrett, 1971; Meudell, 1972; Murray \& Newman, 1973; Salthouse, 1974) used the retroactive interference paradigm to assess directly the presence and independence of visual and verbal encoding of specific stimulus dimensions. Subjects were typically required to recall the identity of letters and/or the location of letters in a matrix. Recall of the stimulus information usually followed the performance of either an auditory or a visual intervening activity. In general, the patterns of recall observed under these experimental conditions have indicated that letter location is primarily encoded visually and letter identity is primarily encoded verbally. It has also been concluded that these modes of representation

The authors wish to express their appreciation to Laura Kilgore for her assistance in testing the subjects. We would also like to thank Bruce Bloxom for his recommendations regarding the data analysis procedures. Requests for reprints should be sent to Terry W. Allen, Department of Psychology, Vanderbilt University, Nashville, Tennessee 37240. are partially independent due to the selective nature of the interference.

Clayton and Warren (1976), in a recent examination of the retroactive interference paradigm, argued that this paradigm has not been used appropriately in studying modality-specific representation. In using this paradigm, the experimenter must make comparisons that distinguish between general interference effects and modality-specific effects produced by the intervening activity. Clayton and Warren noted that previous investigators failed to make the appropriate comparisons, since all analyses compared performance under one intervening activity (e.g., auditory) with performance under a different intervening activity (e.g., visual) in a specified presentation condition (e.g., visual stimulus presentation). Consequently, it is not apparent whether subsequent performance differences were due to differences in the amount of modality-specific interference or the amount of general interference produced by the two intervening activities. In order to solve this problem and assess for modality-specific interference, Clayton and Warren proposed that the modality of the intervening activity be held constant while the modality of presentation or stimulus dimension be systematically varied. The four previously mentioned studies failed to make such comparisons, even though the experimental conditions required to make the comparisons were available (den Heyer \& Barrett, 1971; Meudell, 1972; Murray \& Newman, 1973; Salthouse, 1974).

An additional shortcoming noted by Clayton and Warren (1976) concerns the failure to test for differences in the degree of original learning of location and identity information. The experimental condition necessary to test for differences in the degree of original learning-an unfilled retention interval-was not included in the design of the Meudell (1972) study. The conclusions drawn in the other three previously 
mentioned studies (den Heyer \& Barrett, 1971; Murray \& Newman, 1973; Salthouse, 1974) are also ambiguous, since the results were potentially confounded with degree of original learning. Finally, Murray and Newman (1973) used performance scores for identity and location information that were not independent of each other. Consequently, Clayton and Warren concluded that neither visual mode-specific interference nor selective visual interference with spatial location have yet been unambiguously demonstrated.

The purpose of the present study was to assess the presence of both visual and verbal representation modes by using a design and procedure that would correct for the problems noted by Clayton and Warren (1976). It was hypothesized that if location information is stored visually, then the recall of this information would be more susceptible to interference from a visual interpolated activity. Likewise, if identity information is stored verbally, then the recall of this information will be more susceptible to interference from a verbal interpolated activity. In addition, two other issues were examined by this study. First, a kinesthetic intervening activity was included to examine the effects of a nonvisual and nonverbal activity on performance. Second, the spatial arrangement of the stimulus array was systematically varied in order to assess the effects of stimulus context on the mode of representation.

\section{METHOD}

\section{Subjects}

The subjects were 34 adults recruited from undergraduate psychology classes. The final sample, however, was composed of 30 subjects ( 15 males and 15 females), since the data for 4 subjects were excluded due to experimenter error in the presentation of the task.

\section{Apparatus \\ Matrix stimuli were rear-projected onto a translucent projection screen by a Kodak carousel slide projector. A Lafayette tachistoscopic shutter was attached to the lens of the projector; the shutter speed was set for $.5 \mathrm{sec}$. Presentation of a matrix was subject initiated by the press of a button that triggered the shutter. The subject recalled the letters and their positions in the matrix by writing them on a sheet of paper containing a blank mimeographed matrix in the center. \\ Visual interpolated stimuli were rear-projected onto a second translucent projection screen by a Kodak carousel slide projector, and auditory interpolated stimuli were presented by means of a cassette tape recorder. The kinesthetic interpolated stimuli were presented behind a black cloth screen. A Hunter automatic timer was used to measure the $10-\mathrm{sec}$ interpolated activity interval. A metal box with two pushbuttons labeled "even" or "odd" was used by the subject during the visual and auditory interpolated activities.}

\section{Stimuli}

Two types of matrix stimuli were used, a 1 by 6 matrix and a 2 by 3 matrix. The projected sixe of the 1 by 6 matrix was approximately $27.9 \times 4.5 \mathrm{~cm}$, and the projected size of the 2 by 3 matrix was approximately $14.0 \times 9.5 \mathrm{~cm}$. Twenty slides were made of each type of matrix. A matrix contained 2 of the following 10 uppercase letters: I, E, O, U, P, F, C, J, H, and L. The projected size of each letter was approximately $2.5 \mathrm{~cm}$.
The following counterbalancing constraints were used to determine the arrangement of the letters in each set of 20 slides. Each letter was used at least four times (twice during the first 10 trials and twice during the second 10). A letter was not reused in either the same matrix or in the immediately adjacent matrices. Also, a given pair of letters (e.g., E-O) appeared only once throughout the entire 40 trials of the experiment. Analogous counterbalancing constraints were used to determine the positions of the letters in the matrix. For example, two letters were not allowed to appear in adjacent cells either horizontally or vertically.

Stimuli for the auditory interpolated activity consisted of two-digit numbers read from a table of random numbers and recorded on a cassette tape. These numbers were presented at a rate of about $1 / \mathrm{sec}$. Stimuli for the kinesthetic interpolated activity consisted of 19 randomly selected plastic numbers from 1 to 40 . The numbers (each about $5.1 \mathrm{~cm}$ tall) were attached to the center of a $10.2 \times 10.2 \mathrm{~cm}$ square of cardboard. The presentation rate during the kinesthetic interpolated activity was subject controlled, since each person was instructed to work as quickly as possible. Finally, stimuli for the visual interpolated activity included 40 black-and-white randomly ordered slides of the numbers 1 to 40 . The projected size of each was approximately $2.9 \mathrm{~cm}$. The presentation rate for the visual interpolated stimuli was approximately $1 / \mathrm{sec}$.

\section{Procedure}

The subject was seated in a swivel chair in front of a table, on top of which was located the projection screen for the matrices; the screen was approximately $61 \mathrm{~cm}$ from the subject. Whenever ready, the subject pressed a button on the table, which resulted in a matrix being displayed for $.5 \mathrm{sec}$. The subject's task was to remember both the letters in the matrix and the cells (locations) in which the letters appeared. After the matrix was flashed on the screen, the subject quickly turned his chair $90 \mathrm{deg}$ to his left so that he was seated in front of another table. At this point, he either performed one of the interpolated activities (visual, auditory, or kinesthetic) or engaged in covert rehearsal of the to-be-remembered matrix (unfilled interval condition). There was approximately a 1- to $2-\sec$ interval between stimulus offset and the onset of the interpolated activity. Ten seconds later, upon signal by the experimenter, the subject returned to his original position and drew the letters in the appropriate cells of one of the blank matrices. The subject was allowed $10 \mathrm{sec}$ to reproduce the matrix, which proved to be a more than adequate amount of time for every subject. Afterward, the subject placed the paper face down in a pile out of his immediate sight, and whenever ready, pressed the button to expose the next matrix.

For the block of unfilled interval trials, the subject turned to his left after seeing the matrix and stared at the blank interpolated activity projection screen. His instructions were to concentrate on remembering the letters and their positions in the matrix. For a block of trials with the visual or auditory interpolated activity, the subject either looked at or listened to the numbers and indicated whether a number was odd or even by pressing the appropriately labeled button on the button box. During the kinesthetic interpolated activity, the subject reached under the cloth screen, felt (with both hands) the top number of the pile, and sorted the odd numbers to his left and the even numbers to his right. Subjects were asked, for each of the three interpolated activities, to try to make as few mistakes as possible. One practice trial with a randomly selected matrix was performed prior to the first block of unfilled interval trials. In addition, the subject was given practice with each interpolated activity prior to the block of five trials for that activity. After the experiment was concluded, the subject was asked if he had any special way of remembering the information in the 1 by 6 and 2 by 3 matrices.

Each subject performed 40 trials, a set of 20 with the 1 by 6 
matrix and a set of 20 with the 2 by 3 matrix. The order in which the subject received a set of matrices was randomly varied so that half of the subjects received the 1 by 6 matrix first and half the 2 by 3 matrix first. There were four blocks of five trials within each set of 20 matrices, each block corresponding to matrices recalled in conjunction with an unfilled interval, a visual interpolated activity, auditory interpolated activity, or kinesthetic interpolated activity. The block of five trials with the unfilled interval was always performed first, while the order of the blocks of trials with the interpolated activities was counterbalanced among the subjects. Ten of the subjects received a given interpolated activity first (e.g., visual). Of these 10 subjects, 5 received that interpolated activity followed by one combination of the other two interpolated activities (e.g., visual, auditory, kinesthetic), whereas the remaining 5 received the other possible combination (e.g., visual, kinesthetic, auditory). The order of the blocks of interpolated activity trials was systematically counterbalanced with the order of matrix presentation. Finally, the ordering of the 20 slides within each type of matrix was reversed for half of the subjects so that the first slide seen by these subjects had been the last slide seen by the other subjects.

\section{RESULTS}

Separate location and identity scores were calculated for each subject on the basis of how many elements he recalled correctly per trial. Location was defined as the position of an element in the matrix, and identity was defined as the letter of the alphabet represented by an element in the matrix. On any given trial, a subject could recall a maximum of two correct locations and two correct identities. For example, a subject who recalled two incorrect letters in the wrong positions received a location score of zero and an identity score of zero for that trial. Likewise, if the subject drew the incorrect letters in the correct positions, he received an identity score of zero and a location score of two. Finally, if a subject drew the appropriate letters in the two correct positions, but reversed the order of the letters, he was assigned a location score of two and a letter score of two (this sort of reversal occurred infrequently). A percent-correct score was then calculated for every subject on the set of five trials comprising each memory condition. Each subject, therefore, contributed four location percent-correct scores and four identity percent-correct scores to the analysis. The main findings of this analysis are represented in Table 1.

\section{2 by 3 Matrix}

An analysis of variance was initially performed on the 2 by 3 matrix data with order of matrix presentation, order of interpolated activity presentation, and sex as between-subjects factors and memory condition and stimulus as within-subjects factors. There were no main effects due to order of matrix presentation, order of interpolated activity presentation, or sex of subject. A significant interaction effect occurred between sex of subject and order of matrix presentation $[F(1,18)=5.514, p<.03]$. Male subjects performed worse than female subjects when the 2 by 3 matrix was presented first, but better than female subjects when the 1 by 6 matrix was presented first. In addition, a significant Order of Interpolated Activity by Memory Condition effect occurred $[F(6,54)=2.367, \mathrm{p}<.05]$. In general, subjects performed least accurately during the kinesthetic memory condition except when the kinesthetic condition occurred first. Two significant main effects were also obtained involving memory condition $[F(3,54)=16.994, p<.001]$ and stimulus $[F(1,18)=18.774, \quad p<.001]$. Newman-Keuls comparisons for the main effect of memory condition indicated that performance during the unfilled interval condition was better than each of the other memory conditions $(p<.01)$, and that performance accuracy did not differ among the remaining memory conditions. The significant main effect of stimulus indicated that identity information was recalled more accurately than location information. Most importantly, though, a significant interaction effect was observed between memory condition and stimulus $[\mathrm{F}(3,54)=18.551$, $\mathrm{p}<.001]$. Subjects' recall of identity information was always better than their recall of location information except during the auditory memory condition.

In order to assess for the presence of modalityspecific interference, planned comparisons were made among the mean performances of these conditions in accordance with the suggestions of Clayton and Warren (1976). The appropriate orthogonal comparisons were made using $t$ ratios. The results of these comparisons are listed in Table 1. The comparison of recall during the unfilled interval confirmed that there were no differences between the degree of original learning for location and identity information. The

Table 1

Mean Percent-Correct Scores for Location and Identity as a Function of the Type of Memory Condition

\begin{tabular}{|c|c|c|c|c|c|c|c|c|c|c|}
\hline \multirow{3}{*}{$\begin{array}{l}\begin{array}{c}\text { Memory } \\
\text { Condition }\end{array} \\
\text { Unfilled } \\
\text { Auditory } \\
\text { Visual } \\
\text { Kinesthetic }\end{array}$} & \multicolumn{5}{|c|}{2 by 3 Matrix } & \multicolumn{5}{|c|}{1 by 6 Matrix } \\
\hline & \multicolumn{2}{|c|}{$\begin{array}{l}\text { Stimulus } \\
\text { Location }\end{array}$} & \multicolumn{2}{|c|}{$\begin{array}{l}\text { Stimulus } \\
\text { Identity }\end{array}$} & \multirow{2}{*}{$\begin{array}{c}\begin{array}{c}t \\
(\mathrm{df}=29)\end{array} \\
<1^{*} \\
2.87^{* *}\end{array}$} & \multicolumn{2}{|c|}{$\begin{array}{l}\text { Stimulus } \\
\text { Location }\end{array}$} & \multicolumn{2}{|c|}{$\begin{array}{c}\text { Stimulus } \\
\text { Identity }\end{array}$} & $\frac{(\mathrm{df}=29)}{<1^{*}}$ \\
\hline & $\begin{array}{l}96.0 \\
89.7 \\
73.7 \\
75.3\end{array}$ & $\begin{array}{l}(.71) \\
(1.14) \\
(1.66) \\
(1.78)\end{array}$ & $\begin{array}{l}97.7 \\
81.3 \\
88.7 \\
94.7\end{array}$ & $\begin{array}{l}(.42) \\
(1.50) \\
(1.26) \\
(1.15)\end{array}$ & & $\begin{array}{l}93.3 \\
85.3 \\
74.3 \\
75.3\end{array}$ & $\begin{array}{l}(.83) \\
(1.18) \\
(1.56) \\
(1.73)\end{array}$ & $\begin{array}{l}95.3 \\
75.7 \\
90.3 \\
91.3\end{array}$ & $\begin{array}{l}(.76) \\
(2.07) \\
(1.30) \\
(1.26)\end{array}$ & $\begin{aligned}<1^{*} \\
3.12 * * \\
5.16 \dagger \\
5.16 \dagger\end{aligned}$ \\
\hline
\end{tabular}

Note-Standard deviations are given in parentheses.

*Nonsignificant ** $p<.01 \quad$ tp $<.001$ 
results of the remaining comparisons indicated that the auditory interpolated activity produced greater interference with the recall of letter identity than with letter location, and that the visual interpolated activity produced greater interference with the recall of letter location than with letter identity. Unexpectedly, the kinesthetic intervening activity interfered more with the recall of location than with the recall of identity information.

\section{1 by 6 Matrix}

An analysis of variance that included the same factors used to analyze the 2 by 3 matrix data was performed on the percent-correct scores for the 1 by 6 matrix. The results of this analysis indicated a significant main effect of memory condition $[F(3,54)=11.828$, $p<.001]$. The Newman-Keuls analysis again revealed that performance in the unfilled interval condition was more accurate than in each of the remaining conditions $(\mathrm{p}<.01)$. The other comparisons were not significant. Once again, a significant main effect of stimulus was found $[F(1,24)=27.382, p<.001]$, in which letter identity was recalled more accurately than letter location. A significant Memory Condition by Stimulus interaction effect was also observed $[F(3,72)=9.593, p<.001]$. The pattern of results was the same as that observed for the 2 by 3 matrix. In order to assess for mode-specific interference, $t$ ratios were used to make orthogonal comparisons among the relevant means. The results of these comparisons, indicated in Table 1, are exactly parallel to those reported for the 2 by 3 matrix.

\section{Analysis of Context Effects}

The results of the previous analyses indicated that subjects in both matrix conditions tended to visually encode location information and verbally encode letter identity. It would seem, then, that altering the spatial arrangement of the matrix had no effect on the pattern of stimulus coding used by subjects. Although the spatial arrangement alteration may not have exerted a strong effect on the pattern of coding used by subjects, it still may have affected subjects' representation patterns, but not in a consistent direction. Thus, previous analyses may not have detected such changes.

A correlational analysis of the data obtained from the auditory and visual interference conditions was performed in order to provide a more sensitive indicator of the context manipulation. The presence or absence of a significant correlation would provide an index of the homogeneity of the previously described pattern of stimulus encoding during each matrix condition. A difference score was calculated by subtracting the percent-correct score for recall of location from the percent-correct score for the recall of identity. Difference scores were obtained for performance during both the visual and the auditory interference conditions. The correlation between the two difference scores should not be significant if most or all of the subjects visually encoded location information and verbally encoded identity information. However, a significant correlation would be obtained if a significant number of subjects were employing other patterns of stimulus encoding (e.g., the visual or verbal encoding of both stimulus dimensions or verbal encoding of location and the visual encoding of identity). The results of this analysis did not indicate a significant relationship between these scores in the 2 by 3 matrix condition $(r=.15, p<.10)$. Therefore, the combined results of the correlational analysis and the analysis of variance for the 2 by 3 matrix suggest that individuals predominantly encoded location information visually and identity information verbally.

The results of the correlational analysis performed on the difference scores of the 1 by 6 matrix revealed a significant correlation $(r=.37, p<.05)$. This correlation indicated a more heterogeneous pattern of encoding among subjects. Although there was still a tendency to encode identity and location in a manner similar to the 2 by 3 matrix (as indicated by the comparisons), it appeared that the 1 by 6 matrix was effective in modifying the consistency of patterns of encoding across subjects. For example, it is possible that the arrangement of items in the 1 by 6 matrix (i.e., two letters located in two of six horizontally arranged cells) facilitated the use, by some subjects, of a verbal counting strategy for the recall of location information.

The comparison of the split-half reliability coefficients (corrected for attenuation) computed for the 2 by $3(r=.54)$ and 1 by $6(r=.58)$ matrices revealed no significant difference. These small reliability coefficients appear to be due to the relatively few items in a condition and a restricted range of performance. In this respect, both the reliability and the correlation coefficients tended to reflect the homogeneity of stimulus coding patterns observed across memory conditions.

\section{DISCUSSION}

The results of the present study provide direct evidence for the presence of the visual encoding of letter location and the verbal encoding of letter identity. These results appear, then, to provide the first clear demonstration of visual interference with location information, as well as the presence of a dual pattern of modality encoding.

As indicated by the results of correlation analyses, an individual's choice of a pattern of encoding also appeared to be influenced by the context in which the information was presented. Individuals consistently used the dual pattern of stimulus encoding when the 2 by 3 matrix was presented. Although there was a tendency to use this encoding pattern with the 1 by 6 matrix, presentation of this matrix was somewhat effective in changing patterns of encoding. This result 
would appear to argue against theories of representation that propose that the modality of task presentation or the verbal-nonverbal nature of the stimuli are the sole determinants of the mode of representation used by an individual. Rather, it is felt that the previously mentioned stimulus and task factors interact to influence the individual's perception of the stimulus information and the subsequent choice of mode(s) of representation.

An additional finding of interest concerns the selective interference of location information produced by the kinesthetic interpolated activity. A primary purpose of previous research has been to provide evidence for the existence of a visual short-term memory store (e.g., Meudell, 1972; Salthouse, 1974). The observation of visual interference with recall of stimulus location is consistent with this hypothesis. If one assumes that the modality of presentation determines the mode of representation in short-term memory (as many of these investigators have), then the selective interference of location information by the kinesthetic activity becomes problematic. That is, if one assumes that individuals are encoding the stimulus information presented during the intervening activity in the explicit mode used to present the task, then one would be tempted to conclude that subjects had kinesthetically encoded the location of letters. It might be, then, that individuals use either kinesthetic or visual codes to represent location information. Staying within this conceptual framework, another interpretation of the interference effect would be to maintain that the mode of interference is visual in both instances. Pick (1974) proposed that the visual system becomes specialized in its ability to process information that has a spatial quality. He hypothesized that spatial information obtained through the other sensory modalities is recoded and represented in visual form. In the present task, then, subjects might have recoded the kinesthetic location information during the intervening activity into a visual form. Consequently, this aspect of the kinesthetic condition would provide visual interference with the recall of visually encoded location information.

An alternative and preferred interpretation of the kinesthetic interference effect would be to maintain that the source of interference is spatial rather than modal. In a series of experiments, Shepard and associates (Cooper \& Shepard, 1973; Shepard \& Metzler, 1971) reported that individuals are capable of constructing a mental image with a spatial structure and operating on this internal representation. The internal structure of this representation corresponded in an abstract sense to the spatial structure of the external visual stimulus. Kosslyn $(1975,1976)$ reported similar results in an analysis of the information contained in mental images. Moreover, Kosslyn showed that information represented in the image has a unique internal structure when compared with other forms of representations. From this viewpoint, the findings of the present study can be interpreted in the following manner.

Subjects tended to construct an internal representation of the location of letters in a matrix with the appropriate spatial structure. The visual interpolated activity produced interference with the recall of letter location because the performance of this activity required that subjects utilize the same processes that constructed and maintained the to-be-remembered spatial information. Likewise, the same processes were used in the performance of the kinesthetic activity to construct representations of these stimuli. Interference was produced, then, because the same process involved in constructing and maintaining the internal spatial structure of the visually presented location information was again used by subjects in the performance of the kinesthetic interpolated activity. Thus, in contrast to the previous explanation, the sensory modality in which either the original or interpolated information is presented would not be viewed as the important factor. Rather, the critical factor would concern the degree to which processing the information presented in the two tasks would involve sharing the same processes used to construct and maintain the internal structure of the representation.

\section{REFERENCES}

Clayton, K., \& Warren, M. Methodological problems with the use of the retroactive interference design to infer what is stored. Memory \& Cognition, 1976, 4, 237-243.

Cooper, L. A., \& Shepard, R. N. Chronometric studies of mental images. In W. G. Chase (Ed.), Visual information processing. New York: Academic Press, 1973.

DEn Heyer, K., \& Barrett, B. Selective loss of visual and verbal information in STM by means of visual and verbal interpolated tasks. Psychonomic Science, 1971, 25, 100-102.

Kosslyn, S. M. Information represented in visual images. Cognitive Psychology, 1975, 7, 341-370.

KossLy, S. M. Can imagery be distinguished from other forms of internal representation? Evidence from studies of information retrieval times. Memory \& Cognition, 1976, 4, 291-297.

Meudell, P. Short-term visual memory: Comparative effects of two types of distraction on the recall of visually presented verbal and nonverbal material. Journal of Experimental Psychology, 1972, 94, 244-247.

MurRay, D., \& Newman, F. Visual and verbal coding in short-term memory. Journal of Experimental Psychology, $1973,100,58-62$.

PICK, H. L., JR. Visual coding of nonvisual spatial information. In R. B. MacLeod \& H. L. Pick, Jr. (Eds.), Perception: Essays in honor of James J. Gibson. Ithaca: Cornell University Press, 1974.

SALthouse, T. Using selective interference to investigate spatial memory representations. Memory \& Cognition, 1974, 2, 749-757.

SHEPARD, R. N., \& Metzler, J. Mental rotation of threedimensional objects. Science, 1971, 171, 701-703.

SPERLING, G. A model for visual memory tasks. Human Factors, 1963, 5, 19-31.

Sperling, G. Successive approximations to a model for short-term memory. Acta Psychologica, 1967, 27, 285-292.

(Received for publication June 3, 1977; accepted October 21, 1977.) 